\title{
Miranda
}

Revue pluridisciplinaire du monde anglophone /

Multidisciplinary peer-reviewed journal on the English-

speaking world

5 | 2011

South and Race / Staging Mobility in the United States

\section{Symbiose et circulation dans of Mice and Men de John Steinbeck}

\section{Damien Alcade}

\section{OpenEdition}

Journals

\section{Electronic version}

URL: http://journals.openedition.org/miranda/2541

DOI: $10.4000 /$ miranda.2541

ISSN: 2108-6559

Publisher

Université Toulouse - Jean Jaurès

\section{Electronic reference}

Damien Alcade, "Symbiose et circulation dans Of Mice and Men de John Steinbeck", Miranda [Online], 5 | 2011, Online since 29 November 2011, connection on 16 February 2021. URL: http:// journals.openedition.org/miranda/2541 ; DOI: https://doi.org/10.4000/miranda.2541

This text was automatically generated on 16 February 2021.

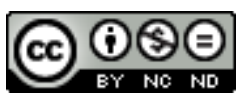

Miranda is licensed under a Creative Commons Attribution-NonCommercial-NoDerivatives 4.0 International License. 


\title{
Symbiose et circulation dans of Mice and Men de John Steinbeck
}

\author{
Damien Alcade
}

1 L'intérêt que portait John Steinbeck à la biologie est indéniable, et doit beaucoup à la relation d'amitié profonde qu'il entretenait avec Ed Ricketts, biologiste marin en Californie, avec qui il avait pris l'habitude de converser pour essayer de révéler les multiples ramifications des différents concepts et idées servant de point de départ à ces discussions. Richard Astro, dans son introduction au carnet de voyage que Steinbeck et Ricketts ont écrit en commun, The Log from the Sea of Cortez, cite quelques passages du texte que Steinbeck écrivit à la suite de la mort de son ami en avril 1948, qui résume bien la démarche évoquée ci-dessus :

In "About Ed Ricketts", Steinbeck recalls that "very many conclusions Ed and I worked out together through endless discussion and reading and observation and experiment. "They had a game, he notes, "which we playfully called speculative metaphysics. It was a sport of looping off a piece of observed reality and letting it move up through the speculative process like a tree growing tall and bushy. We observed with pleasure how the branches of thought grew away from the trunk of external reality. "Indeed, notes Steinbeck, "we worked together, and so closely that I do not now know in some cases who started which line of speculation since the end thought was the product of both minds. I do not know whose thought it was." (sic) (Astro, xvii)

2 Parmi les sujets discutés, la biologie et les théories relatives au sujet tenaient une part prépondérante, en particulier les travaux de deux scientifiques, William Emerson Ritter et Walder Clyde Allee, comme l'explique Richard Astro :

They spent endless hours in Ed's lab discussing the work of Allee and Ritter as Steinbeck worked on his novels and short stories and Ricketts studied what he called "the good, kind, sane little animals," the marine invertebrates of the Central California coast. (Astro, xiii)

3 Ritter est connu pour son concept de superorganisme, qu'il explique en ces termes dans son livre, The Unity of the Organism, or the Organismal Conception :

In all parts of nature and in nature itself as one gigantic whole, wholes are so related to their parts that not only does the existence of the whole depend upon the 
orderly cooperation and interdependence of the parts, but the whole exercises a

measure of determinative control over its parts. (repris dans Astro, xi)

Ce concept est essentiel à une pleine compréhension de l'œuvre de Steinbeck, de même que le sont les travaux de W.C. Allee, en particulier son livre Animal Aggregations, dans lequel il expose ce qu'il appelle le principe de coopération, qu'il définit de la sorte : "an automatic mutual interdependence among organisms" (Allee, 355). Le livre d'Allee recense également les différents types d'agrégations animales que l'on peut trouver dans la nature, et notamment les relations symbiotiques entre deux organismes, un type de relation que nombre de critiques (Brian Leahy Doyle par exemple dans son article "Tragedy and the Non-teleological in Of Mice and Men" paru en 2006) ont cru retrouver dans of Mice and Men, roman de John Steinbeck publié en 1937. George est un petit brun vivace, tandis que Lennie est un géant simple d'esprit, et ce couple singulier fonctionne de telle manière qu'il est possible d'y voir une application du concept de symbiose à deux individus humains, pour signifier l'étroite interdépendance qui semble unir les deux êtres. Nous allons essayer d'étudier cette notion de couple symbiotique, et voir comment la forte interdépendance de ces deux individus est directement liée à leur circulation de ranch en ranch, circulation dont nous essaierons d'étudier les modalités et la dynamique, en nous appuyant sur les travaux de Ritter, d'Allee, de Charles Darwin mais aussi sur le concept d'animal politique d'Aristote, qui révèle un axe de lecture potentiel pour le roman.

\section{Lennie et George comme couple symbiotique}

Donnons tout d'abord une définition de la symbiose, telle qu'elle est décrite dans le Chambers Biology Dictionary: "An intimate partnership between two organisms, in which the mutual advantages normally outweigh the disadvantages." Cette définition moderne est restrictive, et doit être nuancée, car les avis diffèrent sur le fait de ne pas qualifier de symbiotique les phénomènes qui ne sont pas nécessairement bénéfiques aux différentes parties impliquées. Il est possible de distinguer trois principaux types de symbiose, tous référencés dans le livre d'Allee, lorsque l'on considère le terme au sens large :

- le parasitisme, où un seul des deux individus tire profit de l'association, au détriment de l'autre.

- le commensalisme, lorsque l'association ne bénéficie qu'à un seul des deux individus, sans que l'autre soit lésé.

- enfin, le mutualisme, qui est bénéficiaire aux deux parties.

6 Le roman met clairement en évidence un rapport étroit, presque fusionnel, entre Lennie et George. La manière dont les deux personnages sont introduits est à ce titre exemplaire :

For a moment the place was lifeless, and then two men emerged from the path and came into the opening by the green pool. They had walked in single file down the path, and even in the open one stayed behind the other. Both were dressed in denim trousers and in denim coats with brass buttons. Both wore black, shapeless hats and both carried tight blanket rolls slung over their shoulders. (Steinbeck, 1937, 2)

7 Tout d'abord, la première référence aux personnages est plurielle, "two men". La deuxième phrase de l'extrait implique que la configuration du groupe ne dépend pas de l'environnement mais bien d'une organisation interne, et qu'elle semble de fait 
immuable. Le couple avance d'un seul bloc, et l'utilisation de l'expression "in single file" s'avère intéressante en ce qu'elle décrit par une entité unique ("a single file") un couple d'individus. C'est également un des effets de la répétition du pronom "both" pour décrire les deux hommes dans la phrase suivante. Rappelons que "both" fait partie du système du duel en anglais, comme "either" ou "neither", un système qui se démarque du pluriel en ce qu'il désigne la totalité d'un ensemble à deux éléments, tandis que le pluriel fait référence à une multiplicité d'objets. Avec l'utilisation de "both" plutôt que "they", qui aurait été possible avec la même syntaxe, c'est l'importance du couple considéré comme un ensemble qui est mise en avant. On voit ainsi comment la matière du texte amène à considérer le couple Lennie/George comme une seule entité dès le début du roman. Les deux individus qui composent ce couple sont différents, mais malgré tout Lennie n'est décrit que dans son rapport avec George dans ce premier chapitre : il est l'opposé de George (Steinbeck, 1937, 2), il est celui qui suit (Steinbeck, 1937, 3), il est le compagnon (Steinbeck, 1937, 3). Les mots qui sont utilisés pour décrire Lennie ne prennent sens que parce qu'il est accompagné de quelqu'un. Ce rapport étroit entre les deux hommes est également mis en avant lorsqu'ils discutent du rêve qu'ils ont d'obtenir ensemble une ferme dont ils pourraient s'occuper. Ce qui différencie Lennie et George des autres travailleurs réside dans le rapport qu'ils entretiennent, et l'importance de ce rapport est mise en avant par l'utilisation d'italiques lorsque George raconte le rêve à Lennie pour la première fois :

George went on. "With us it ain't like that. We got a future. We got somebody to talk to that gives a damn about us. We don't have to sit in no bar room blowin' in our jacks jus' because we got no place else to go. If them other guys gets in jail they can rot for all anybody gives a damn. But not us."

Lennie broke in. "But not us! An' why? Because... because I got you to look after me, and you got me to look after you, and that's why." (Steinbeck, 1937, 15)

Lennie est également décrit tout au long du roman comme une extension du corps et de la volonté de George. Dès le premier chapitre, il essaie d'imiter George autant qu'il le peut :

Lennie, who had been watching, imitated George exactly. He pushed himself back, drew up his knees, embraced them, looked over to George to see whether he had it just right. He pulled his hat down a little more over his eyes, the way George's hat was. (Steinbeck, 1937, 4)

Lorsque George lui demande de faire quelque chose, c'est moins sa volonté que son corps qui répond à l'injonction, comme lorsque George demande à Lennie de lui rendre la souris morte qu'il garde avec lui :

"Give it here!" said George.

"Aw, leave me have it, George."

"Give it here!"

Lennie's closed hand slowly obeyed. (Steinbeck, 1937, 6)

10 Cette relation de complète dépendance a amené Lennie à se jeter dans une rivière sur l'ordre de George au risque de se noyer, dans une anecdote que ce dernier raconte à Slim, un autre travailleur, ce qui montre à quel point il est asservi à George, à quel point il dépend de lui. George répète plusieurs fois que Lennie ne pourrait même pas se nourrir sans lui :

"Well. I could. I could go off in the hills there. Some place I'd find a cave."

"Yeah? How'd you eat. You ain't got sense enough to find nothing to eat."

(Steinbeck, 1937, 14) 
11 Cet aspect nourricier de George rapproche la relation entre Lennie et George d'un phénomène de symbiose au sens strict, l'un des individus au moins ne semblant pas pouvoir survivre sans être associé à l'autre. Mais s'il est possible de considérer la relation qui unit George et Lennie comme une transposition sur le mode symbolique du phénomène de symbiose à deux individus humains, à l'aune des travaux critiques sur le sujet et des multiples exemples qui émaillent le texte et qui mettent l'accent sur le rapport étroit qui unit les deux individus, il faut maintenant se demander de quel type de relation symbiotique il s'agit.

$12 \mathrm{Au}$ premier abord, la relation entre Lennie et George semble se rapprocher d'une relation parasitique, Lennie fonctionnant comme parasite de l'hôte George. En effet, ce dernier semble pâtir de son association avec Lennie, comme il aime à le répéter lorsqu'il est en colère :

George went on furiously. "I got you! You can't keep a job and you lose me ever' job I get. Jus' keep me shovin' all over the country all the time. An' that ain't the worst. You get in trouble. You do bad things and I got to get you out." (Steinbeck, 1937, 12)

Il n'est pas nécessaire d'étoffer cette idée selon laquelle Lennie pose des problèmes à George, puisqu'elle est assez évidente au vu de la trame narrative. Que la relation entre Lennie et George soit profitable à Lennie ne fait pas de doute non plus. Il n'est pas nécessaire non plus de s'attarder sur une éventuelle relation commensaliste entre les deux individus, car il est certain que la vie de George est transformée par la présence de Lennie. En revanche, il apparaît que d'une certaine manière George profite de la relation qu'il entretient avec Lennie, au point que les avantages mutuels de cette relation dépassent les désavantages évoqués, pour reprendre la définition de la symbiose donnée précédemment. Il serait alors possible de voir dans la relation entre Lennie et George une relation mutualiste, dont il va s'agir d'étudier les modalités, car c'est dans cette relation de bénéfice mutuel que symbiose et circulation entrent en dialogue.

\section{L'intérêt de George ou le couple symbiotique en mouvement}

14 George explique la manière dont sa relation avec Lennie a débuté lorsqu'il s'entretient avec Slim, un personnage qui garde tout le long du roman un statut de confident pour George :

“It ain't so funny, him an' me goin' aroun' together," George said at last. "Him and me was both born in Auburn. I knowed his Aunt Clara. She took him when he was a baby and raised him up. When his Aunt Clara died, Lennie just come along with me out workin'. Got kinda used to each other after a little while." (Steinbeck, 1937, 45)

C'est le principe de coopération d'Allee qui est illustré ici, où deux individus s'associent de manière quasiment automatique. De cette relation découle l'apprentissage par George d'un certain sens moral, d'une sympathie envers Lennie :

One day a bunch of guys was standin' around up on the Sacramento River. I was feelin' pretty smart. I turns to Lennie and says, 'Jump in.' An' he jumps. Couldn't swim a stroke. He damn near drowned before we could get him. An' he was so damn nice to me for pullin' him out. Clean forgot I told him to jump in. Well, I ain't done nothing like that no more. (Steinbeck, 1937, 45-46) 
Ce changement dans l'attitude de George marque le développement d'un sens moral qui est le fruit d'un lien social, un développement qui est aussi évoqué par Crooks, le palefrenier qui vit à l'étable :

A guy sets alone out here at night, maybe readin' books or thinkin' or stuff like that. Sometimes he gets thinkin', an' he got nothing to tell him what's so an' what ain't so. Maybe if he sees something', he don't know whether it's right or not. He can't turn to some other guy and ask him if he sees it too. He can't tell. He got nothing to measure by. (Steinbeck, 1937, 82-83)

17 Cet apprentissage de la morale par la présence de l'autre rappelle la notion Darwinienne d'instinct de sympathie. L'influence de Darwin sur Steinbeck a été relevée par la critique - notamment dans le livre de Brian E. Railsback Parallel Expeditions: Charles Darwin and the Art of John Steinbeck - et on peut voir dans la description d'un certain sens moral acquis par le biais d'une relation d'amitié avec un autre individu une illustration de ce que Darwin explique dans son livre Descent of Man :

The development of the moral qualities is a more interesting problem. The foundation lies in the social instincts, including under this term the family ties. These instincts are highly complex, and in the case of the lower animals give special tendencies towards certain definite actions; but the more important elements are love, and the distinct emotion of sympathy. Animals endowed with the social instincts take pleasure in one another's company, warn one another of danger, defend and aid one another in many ways. These instincts do not extend to all the individuals of the species, but only to those of the same community. [...] Sympathy, though gained as an instinct, is also much strengthened by exercise or habit. (Darwin, 324)

Le fait que George ait laissé Lennie venir travailler avec lui après la mort de sa tante Clara est l'illustration d'un instinct de sympathie rudimentaire, que la quasi-noyade de Lennie a servi à renforcer. George tire plaisir d'être accompagné par Lennie, comme il le dit lui-même à Slim, servant une fois encore de confident: "It's a lot nicer to go around with a guy you know" (Steinbeck, 1937, 39). George est également conscient que c'est sa relation avec Lennie qui fait qu'il reste quelqu'un de bon :

I ain't got no people, George said. I seen the guys that go around on the ranches alone. That ain't no good. They don't have no fun. After a long time they get mean. They get wantin' to fight all the time. (Steinbeck, 1937, 46)

Voici donc un des premiers bénéfices que George tire de sa relation avec Lennie : guidé par son instinct de sympathie, il acquiert et conserve un sens moral qui fait défaut aux solitaires.

20 Le deuxième bénéfice que George tire de sa relation avec Lennie, peut-être le plus important, est son obligation à circuler de ranch en ranch, à se déplacer. George s'en plaint, comme dans l'exemple cité plus haut: 'Jus' keep me shovin' all over the country all the time" (Steinbeck, 1937, 12). Dans un accès de colère, il se laisse aller à imaginer quelle serait sa vie sans Lennie :

God a'mighty, if I was alone I could live so easy. I could go get a job an' work, an' no trouble. No mess at all, and when the end of the month come I could take my fifty bucks and go into town and get whatever I want. Why, I could stay in a cat house all night. I could eat any place I want, hotel or any place, and order any damn thing I could think of. An' I could do that every damn month. Get a gallon of whisky, or set in a pool room and play cards or shoot pool. (Steinbeck, 1937, 12)

21 Il est intéressant de constater à quel point cette utopie relève de la stase. Sans Lennie, George serait condamné à répéter le même schéma, sans jamais en changer. De la 
même manière, Lorsque Lennie menace George de le quitter, son alternative relève également de la stase :

If you don't want me, you only jus' got to say so, and I'll go off in those hills right there - right up in those hills and live by myself. An' I won't get no mice stole from me. (Steinbeck, 1937, 14)

Il est important de savoir à quel point l'absence de mouvement dans les œuvres de Steinbeck traitant de la Grande Dépression est quelque chose de négatif. Il suffit pour s'en convaincre de lire The Grapes of Wrath avec par exemple le personnage de Muley Graves qui refuse de quitter les terres désolées de l'oklahoma et dont le nom et l'attitude portent des connotations négatives évidentes. Se déplacer, circuler dans l'espace américain est une nécessité, synonyme de survie pour les migrants de l'oklahoma dans The Grapes of Wrath. La circulation de Lennie et George est conditionnée par le fait qu'ils sont ensemble, mais elle leur donne aussi un but, cette petite ferme qu'ils souhaitent acquérir ensemble pour obtenir une indépendance, représentation de l'idéal Jeffersonien de l'agrarianisme cher à Steinbeck, idéal qu'il expose également dans The Grapes of Wrath. Que ce rêve ne se réalise pas n'est pas important, c'est l'éventualité de sa réalisation qui le rend valide, et c'est par leur mouvement de ranch en ranch que Lennie et George s'approchent de ce rêve, puisque c'est en allant dans le ranch servant de décor au roman que Lennie et George rencontrent Candy, le vieux travailleur qui leur propose de s'associer à eux pour rendre possible ce rêve en leur prêtant de l'argent. Pour résumer, l'association entre Lennie et George entraîne un mouvement forcé du couple qui se trouve être quelque chose de positif puisqu'il leur permet d'échafauder un rêve commun pour s'élever au dessus de leur condition, que le hasard des rencontres qu'ils font rend palpable.

En ce sens, on peut dire, si l'on considère comme valide la comparaison de la relation Lennie/George au processus de symbiose, que cette relation symbiotique s'apparente à un phénomène de mutualisme, les deux individus profitant de l'association, dont les bénéfices dépassent les désavantages. Il est aussi essentiel de noter que ce rêve est consubstantiel à l'existence de cette relation, car lorsque Lennie tue la femme de Curley et disparait, il est clair pour George que tous leurs projets communs disparaissent également, même si les conditions de leur réalisation n'ont pas disparu, puisque le vieux Candy est toujours là et peut toujours avancer l'argent :

Now Candy spoke his greatest fear. 'You an' me can get that little place, can't we, George? You an' me can go there an' live nice, can't we, George? Can't we?

Before George answered, Candy dropped his head and looked down at the hay. He knew.

George said softly, "- I think I knowed from the very first. I think I knowed we'd never do her. He usta like to hear about it so much I got to thinking maybe we would."

"Then - it's all off?" Candy asked sulkily.

George didn't answer his question. George said, 'I'll work my month an' I'll take my fifty bucks an' I'll stay all night in some lousy cat house. Or I'll set in some poolroom till ever'body goes home. An' then I'll come back an' work another month an' I'll have fifty bucks more." (Steinbeck, 1937, 107)

Sans cette relation symbiotique, c'est le retour à la stase, un sentiment qui est accentué par la répétition des termes utilisés par George au début du roman pour décrire ce qu'il aurait souhaité en l'absence de Lennie. La maison de passe, le billard, les cinquante dollars - l'écho intratextuel illustre l'état d'immobilité qui frappe un George alors 
coupé de son binôme. Le jeu sur les pronoms dans les dernières paroles entre Lennie et George illustre à ce titre l'importance du couple pour la survie du rêve :

Lennie begged, "Les' do it now. Le's get that place now."

"Sure, right now. I gotta. We gotta." (Steinbeck, 1937, 120)

En passant de "I" à "We", George reconnaît que ce rêve est un rêve commun, qui ne peut pas fonctionner sans ce pluriel essentiel. On retrouve ici un avatar de la théorie du superorganisme de Ritter, puisque c'est le fait qu'ils soient ensemble qui détermine l'objectif à atteindre et qui le rend possible.

Bien qu'une lecture basée sur la biologie semble suffisante pour éclairer en partie la transposition sur le mode symbolique du phénomène de symbiose mutualiste qui unit Lennie et George, il semble également que ce faisant Steinbeck rejoint le concept d'animal politique d'Aristote. Ce rapprochement n'est qu'une piste de réflexion qui mériterait une étude plus poussée. Elle est évoquée ici car elle permet d'intégrer une composante politique à la représentation du couple Lennie/George en tant qu'entité symbiotique, une composante qui s'intègre tout à fait aux préoccupations de Steinbeck à cette époque. Steinbeck était un avide lecteur de philosophes antiques, comme le montre son biographe Jackson J. Benson ainsi que les multiples références à Hérodote, Thucydide et Xénophon qui parsèment ses premières œuvres, mais il manque une étude approfondie qui étudierait en détail les affinités de l'auteur avec la philosophie antique. Il est clair que Steinbeck lisait Aristote puisque selon Robert DeMott, dans son livre Steinbeck's Reading qui recense les livres dont Steinbeck disposait dans sa bibliothèque, ce dernier possédait plusieurs ouvrages du philosophe grec. Cependant, l'influence de ce dernier sur la prose de Steinbeck reste à évaluer en détail. Dans les limites énoncées, il semble que l'on puisse voir dans ce couple symbiotique cherchant à réaliser un rêve symbolisant un idéal de société une application du concept d'animal politique qu'Aristote développe dans son traité Les Politiques.

\section{Aristote et la genèse d'une cité}

Dans son traité sur les politiques, Aristote considère l'homme comme un animal politique. On peut imaginer que le concept ait pu intéresser Steinbeck, lui qui se targuait de considérer l'homme comme une espèce animale avant tout. Dans le second chapitre des Politiques, Aristote explique que la genèse d'une cité provient de l'union de deux individus ne pouvant exister l'un sans l'autre :

\footnotetext{
Ainsi, il est nécessaire tout d'abord que s'unissent les êtres qui ne peuvent exister l'un sans l'autre, par exemple la femme et l'homme en vue de la procréation (et il ne s'agit pas d'un choix réfléchi, mais comme aussi pour les autres animaux et les plantes d'une tendance naturelle à laisser après soi un autre semblable à soi); et celui qui commande et celui qui est commandé, et ce par nature, en vue de leur mutuelle sauvegarde. (Aristote, 87).
}

Le rapprochement avec le phénomène de symbiose tel qu'il est métaphorisé dans of Mice and Men est évident, George étant celui qui commande et Lennie celui qui est commandé. Pour Aristote, de ce couple inséparable naît la famille, puis la communauté formée de plusieurs familles (le village), et enfin la cité qui permet l'accès au bonheur parce qu'elle est en autarcie :

Et la communauté achevée formée de plusieurs villages est une cité dès lors qu'elle a atteint le niveau de l'autarcie pour ainsi dire complète; s'étant donc constituée 
pour permettre de vivre, elle permet une fois qu'elle existe, de mener une vie heureuse. (Aristote, 90) l'importance de l'autarcie pour accéder au bonheur est présente à la fois chez Aristote et dans la petite ferme que les deux travailleurs veulent acquérir, ce qui n'est pas étonnant puisque l'autosuffisance est une notion essentielle dans l'agrarianisme jeffersonien dont Steinbeck se fait le héraut dans of Mice and Men comme dans The Grapes of Wrath. La finalité politique de l'animal humain chez Steinbeck diffère d'Aristote, mais la dynamique est la même : d'un couple dont les individus ne peuvent exister l'un sans l'autre, un couple symbiotique, peut naitre une organisation qui est un modèle de société au niveau politique - la cité pour Aristote, et la ferme indépendante pour Steinbeck. L'étape de la famille est essentielle chez Aristote, mais chez Steinbeck ce n'est pas le cas. Il semble que Steinbeck manifeste une affinité avec Allee ici, puisque ce dernier note dans son ouvrage que la fondation familiale n'est pas toujours nécessaire à l'agrégation d'organismes vivants :

These illustrations suggest the possibility of the formation of groups of decided social integration as the result of the congregation of individuals, without a family aggregation of any sort appearing in this particular type of organization. (Allee, 346)

Ainsi, l'agrégation des individus peut se faire sans le modèle familial comme base, est c'est exactement ce qui se passe dans of Mice and Men. En effet, c'est ici que le mouvement du couple symbiotique Lennie/George prend tout son sens au niveau politique, car ce mouvement entraîne un regroupement, chacun aidant à transformer le rêve en réalité tangible. Ainsi, le vieux Candy vient s'intégrer au groupe, et se faisant transforme le rêve en éventualité, puisqu'il apporte de l'argent. Le palefrenier noir, Crooks, demande également à se joindre à eux :

If you guys would want a hand to work for nothing - just his keep, why I'd come an' lend a hand. I ain't so crippled I can't work like a son-of-a-bitch if I want to. (Steinbeck, 1937, 86-87)

Il demande cela à Lennie, et l'arrivée de la femme de Curley coupe court à la discussion, si bien que Crooks ne s'intègre finalement pas au projet, mais c'est la potentialité de l'intégration de Crooks qui est importante, de même que c'est dans la potentialité de la réalisation du rêve d'indépendance que le rêve trouve sa validité. L'intégration du vieil homme dans le projet de Lennie et George et la possible intégration du palefrenier est à rapprocher du développement d'un sens moral tel que l'entend Darwin :

[Man's] sympathies became more tender and widely diffused, so as to extend to the men of all races, to the imbecile, the maimed, and other useless members of society, and finally to the lower animals. (Darwin, 253)

Ainsi, le mouvement du couple Lennie/George entraîne l'agrégation d'individus dont le statut -- vieillard, minorité ethnique - montre le développement d'un sens moral permettant l'acceptation de la différence, de la même manière que George apprend à accepter et à apprécier la différence de Lennie. Ce mouvement associé à la potentielle agrégation de ces individus entraîne une possible réalisation d'un rêve d'indépendance symbolisant un idéal de société basé sur l'agrarianisme Jeffersonien. La transposition que fait Steinbeck de la dynamique Aristotélicienne de genèse de la cité, en associant ce concept à sa vision biologique de l'homme fondée sur les théories de Allee, Ritter et Darwin, permet de donner à la circulation du couple Lennie/George de ranch en ranch une chose essentielle : un objectif. 
cet objectif ne se réalise pas n'est que secondaire, c'est dans l'éventualité de sa réalisation que réside sa validité. A ce titre, la fin du roman intègre, de manière paradoxale, une pointe d'espoir. En effet, lorsque George a exécuté Lennie pour lui éviter d'être lynché, il se retrouve seul, le pistolet en main. Ce n'est pourtant pas la dernière scène du roman. En effet, le tout dernier événement est l'arrivée des poursuivants, et en particulier de Slim, avec qui George s'est bien entendu durant son séjour au ranch. Il est intéressant de constater que le rapprochement entre Slim et George est clairement mis en avant : "Slim came directly to George and sat down beside him, sat very close to him" (Steinbeck, 1937, 121). La répétition insiste sur ce rapprochement, et Slim touche ensuite le coude de George et l'entraîne avec lui :

slim twitched George's elbow. 'Come on, George. Me an' you'll go in an' get a

drink." [...] He led George into the entrance of the trail and up toward the highway.

(Steinbeck, 1937, 121)

Le rapprochement devient un contact physique, et l'utilisation de "Me an' you" insiste sur la création d'un nouveau binôme, qui quitte l'état de stase pour se mettre en mouvement. A travers ce nouveau couple qui se crée, c'est un espoir qui renaît, le roman ayant démontré comment ce rapprochement entre deux individus est le zygote nécessaire ouvrant le chemin à nombre de potentialités. Comment ne pas voir ici également une autre illustration de l'instinct de sympathie de l'être humain? Et dans l'optique de lecture mise en avant dans le présent article, les deux derniers mots du roman ne sont pas du tout surprenants, et prennent tout leur sens :

Curley and Carlson looked after them. And Carlson said, "Now what the hell ya suppose is eatin' them two guys?" (Steinbeck, 1937, 121)

guys ?", voilà peut-être tout l'enjeu du roman résumé. Deux hommes, et à travers leur mouvement commun un champ de possibilités, que la marque interrogative qui clôture le roman laisse deviner.

\section{Conclusion}

Steinbeck considère donc l'être humain comme un animal, que l'instinct de sympathie et le principe de coopération poussent à s'agréger pour survivre, ce qui développe également son sens moral. Une des spécificités de cet animal, selon Aristote, est d'être un animal politique, dont la finalité est de créer naturellement une société indépendante que Steinbeck relie à l'idéal Jeffersonien d'agrarianisme qui lui est cher, à partir d'un couple interdépendant originel que Steinbeck représente en s'appuyant sur la notion de mutualisme, type de symbiose où les deux individus participant de la relation tirent un bénéfice de celle-ci. La circulation forcée de ce couple de ranch en ranch entraîne une agrégation, réelle ou potentielle, d'individus autour d'eux, rendant l'objectif final du mouvement tangible. Cette transposition d'idées et de théories diverses entre en résonnance avec le processus de métaphysique spéculative entre Steinbeck et Ricketts évoqué en introduction. Of Mice and Men est un roman d'espoir plutôt que d'espoir déçu, espoir qui naît de la coopération entre les individus, thème que l'on retrouvera dans The Grapes of Wrath. Le titre du roman, of Mice and Men, contient déjà en germe beaucoup de ces idées : la mise sur le même plan de l'homme et de l'animal, le pluriel qui évoque le regroupement pour former des plans, comme le montre le poème qui a donné son titre au roman, To a Mouse de Robert Burns : "The best laid schemes o' mice an' men / Gang aft agley". Que ces plans n'aboutissent pas n'est 
pas aussi important pour Steinbeck que le fait qu'ils existent, et c'est à travers la coopération des individus que cette potentialité se fait jour, car elle pousse le couple symbiotique à circuler, à rencontrer et intégrer d'autres personnes, et par là-même à s'approcher de son rêve.

\section{BIBLIOGRAPHY}

Aristote. Les Politiques. Traduit par Pierre Pellegrin. Paris: Flammarion, 1993.

Allee, Walder Clyde. Animal Aggregations. Chicago: The University of Chicago Press, 1931.

Astro, Richards. Introduction to The Log from the Sea of Cortez (1941). London: Penguin Books Ltd, 1995.

Benson, Jackson J. The True Adventures of John Steinbeck, Writer. New York: Viking Press, 1984.

Burns, Robert. The Complete Poems and Songs of Robert Burns. New Lanark: Geddes \& Grosset. 2000.

Darwin, Charles. Evolutionary Writings. New York: Oxford University Press, 2008.

DeMott, Robert. Steinbeck's Reading. New York: Garland Publishing inc, 1984.

Doyle, Brian Leahy. "Tragedy and the Non-teleological in Of Mice and Men." Steinbeck Review 3.2 (2006): 79-86.

Railsback, Brian E. Parallel Expeditions: Charles Darwin and the Art of John Steinbeck. Moscow: University of Idaho Press, 1995.

Ritter, William Emerson. The Unity of the Organism, or the Organismal Conception. Boston : R.G. Badger, 1919.

Rivière, Claude et Larreya, Paul. Grammaire explicative de l'anglais. Paris: Pearson Education France, 2005.

Steinbeck, John. Of Mice and Men (1937). London: Penguin Red Classic, 2006.

--- The Grapes of Wrath (1939), The Viking Critical Library (The Grapes of Wrath: Text and

Criticism, ed. Peter Lisca and Peter Hearle), New York: Penguin Books USA, 1997.

Tanner, Tony. The Reign of Wonder: Naivety and Reality in American Literature. Cambridge:

Cambridge University Press, 1965.

Walker, P.M.B. et al. Chambers Biology Dictionary. Cambridge: Cambridge University Press, 1989.

\section{ABSTRACTS}

Relying on the text, this essay highlights the symbiotic relationship which unites George and Lennie, the two main protagonists in of Mice and Men. It is based on the works of several biologists and naturalists, including Ritter, Allee and Darwin, as well as on Aristotle's political animal theory, which all participate in showing that the mutualistic aspect of this symbiotic relationship comes from the fact that Lennie and George are constantly on the move. 
Cet article évoque, à partir d'exemples tirés du texte, la relation symbiotique qui unit George et Lennie, les deux personnages principaux de of Mice and Men. En s'appuyant sur les travaux de plusieurs scientifiques (Ritter, Allee, Darwin) et sur le concept d'animal politique d'Aristote, il met en avant l'aspect mutualiste de cette relation qui provient principalement de la circulation de ce couple symbiotique dans l'espace américain.

INDEX

Mots-clés: symbiose, circulation, mutualisme

Keywords: symbiosis, circulation, mutualism

\section{AUTHORS}

DAMIEN ALCADE

Doctorant

Université Toulouse 2 - Le Mirail

damien.alcade@univ-tlse2.fr 